\title{
Mechanical Characterization and Influence of the High Temperature Shrinkage of $\beta$-PVDF Films on its Electromechanical Properties
}

\author{
V. SENCADAS, ${ }^{1}$ R. BARBOSA, ${ }^{1}$ J. F. MANO, ${ }^{2}$ \\ and S. LANCEROS-MÉNDEZ ${ }^{1, *}$ \\ ${ }^{1}$ Dept. de Física, Universidade do Minho, 4710-057 Braga, Portugal \\ ${ }^{2}$ Dept. de Eng. de Polímeros, Universidade do Minho, \\ 4800-058 Guimarães, Portugal
}

(Received September 4, 2002; In final form December 15, 2002)

\begin{abstract}
Tensile dynamic mechanical analysis at $1 \mathrm{~Hz}$ was used to characterize the solid rheological properties of $\beta$-PVDF films. Both the elastic and loss moduli and the specific damping capacity were monitored against temperature, allowing the study of the effect of anisotropy upon the viscoelastic properties of the films. The temperature range covered the $\beta$ - and $\alpha$-relaxations. These results are compared to dielectric relaxation results in order to elucidate the electrical and mechanical contributions to the observed relaxations. Further, an important shrinking effect upon heating above $364 \mathrm{~K}$ has been observed, that influences the material properties. This geometrical effect has been monitored by thermal mechanical analysis. The thermal coefficients of linear expansion have been calculated, giving two different regimes for this parameter. The variations at a molecular level have been monitored by FTIR.
\end{abstract}

Keywords: PVDF; dielectric relaxation; mechanical relaxation; infrared

\section{INTRODUCTION}

Ferroelectric polymers are both of great scientific and technological interest. The rapid commercialization of piezoelectric transducers made from these materials also a model for technology transfer. Ferroelectric polymers are a rich system for the study of phase transitions, relaxational processes and ferroelectricity; they exhibit all the interesting physical phenomena associated with inorganic ferroelectrics and yet exhibit fundamentally different microscopic interactions, dominated by the hydrogen bonding and the van der Waals forces [1].

\footnotetext{
*Correspondence address. E-mail: lanceros@ fisica.uminho.pt
} 
Poly(vinilidene fluoride) (PVDF) is the prototypical ferroelectric polymer. PVDF films are polymorphous, containing amorphous and crystalline phases. Films must be textured mechanically and electrically to achieve macroscopic piezoelectric behavior, though they still contain amorphous material and the crystallites are incompletely oriented [2]. The properties of the films strongly depend on the crystallinity and morphology, and these factors suffer significant variations when the material undergoes thermal and mechanical treatments.

PVDF is a linear fluorinated hydrocarbon with a repeat unit $\left(\mathrm{CH}_{2}-\mathrm{CF}_{2}\right)$ of spacing 2.6 $\mathrm{A}$. The PVDF chains have a net dipole moment, pointing from the electronegative fluorine to the electropositive hydrogen, producing a net dipole moment nearly perpendicular to the polymer chain. These chains can crystallize in a quasi-hexagonal close-packed $\beta$-phase structure with dipoles of all chains aligned in a structure with maximum polarization $\mathrm{P} \sim 0.13$ $\mathrm{Cm}^{-2}$. Poling and switching are accomplished by applying a large electric field perpendicular to the chains to reverse the direction of the polarization $[1,3]$.

This polarization is based on the cooperative orientation of the monomer dipoles $\left(\mathrm{CH}_{2}-\mathrm{CF}_{2}\right)$ in ferroelectric domains [3]. In PVDF the melting temperature $T_{M}$ (ranges from 433 to $453 \mathrm{~K}$ ) is lower than the expected Curie temperature $T_{C}$ (calculated in the range 478 to $481 \mathrm{~K}$ ). So, heating the poled $\beta$-PVDF films to temperatures lower than the melting temperature yields an increase of the non-polar $\alpha$-phase and a decrease of the polar $\beta$-phase, but not a complete phase transition and thus not a complete depolarization of the polymer [3].

The ferro-, piezo-, and pyroelectric properties of PVDF are exploited in a number of applications such as sensors of heat, pressure and sound, in which the material may be subjected to severe temperature changes or mechanical stresses [1,3]. An important issue is the dependence of those properties on the structure of PVDF as these properties and even the crystal structure and modification may change with temperature and mechanical stress [3].

In the present investigation both the elastic and loss moduli and the specific damping capacity were monitored against temperature, allowing the study of the effect of anisotropy upon the viscoelastic properties of the films. These mechanical results were compared with previous dielectric results. Further, the shrinking of the material upon heating and its influence in the material properties has been characterized and analyzed for samples with and without electrodes. The variations at a molecular level have been monitored by FTIR. 


\section{EXPERIMENTAL}

The analysed material was a $28 \mu \mathrm{m}$ thick commercial $\beta$-PVDF film from Measurements Specialties, Inc. (Fairfield, NJ, USA). Rectangular samples ( $\sim 3 \mathrm{~mm}$ wide and $20 \mathrm{~mm}$ long) were cut from the original sheet both perpendicular and parallel to the polymeric chains. Longitudinal samples will label the specimens in which the mechanical tests were performed along the stretch direction, whereas transverse samples are for experiments perpendicular to the stretch direction.

Both Dynamical Mechanical Analysis (DMA) and Thermal Mechanical analysis (TMA) experiments were carried out in a Perkin-Elmer DMA7e apparatus using the tensile mode. The DMA experiments were performed at isochronal conditions (frequency of $1 \mathrm{~Hz}$ ), from 223 to $394 \mathrm{~K}$ at a heating rate $2 \mathrm{~K} / \mathrm{min}$. The amplitude of the dynamic stress was $0.1 \mathrm{MPa}$, which was small enough to ensure a linear viscoelastic response from the samples. For the TMA measurements, the deformation of the material was obtained at a temperature rate of $2 \mathrm{~K} / \mathrm{min}$ from 314 to $400 \mathrm{~K}$ in samples with silver electrodes and samples without electrodes.

Infrared spectra were obtained in transmission mode on a BRUKER 66V spectrometer from 400 to $6000 \mathrm{~cm}^{-1}$ using polarised light in perpendicular $(\perp)$ and parallel $(\|)$ modes, with a resolution of $1 \mathrm{~cm}^{-1}$. Samples with the dimensions of $20 \times 10 \mathrm{~mm}^{2}$ were cut and placed in an oven (Carbolite) and heat treated at different temperatures $(334,364,394$ and $414 \mathrm{~K})$ for $\sim 1 \mathrm{~min}$.

\section{RESULTS AND DISCUSSION}

\subsection{Dynamical Mechanical Analysis}

In Fig. 1, the storage modulus $\left(E^{\prime}\right)$ and the loss factor $\left(\tan \delta=E^{\prime \prime} / E^{\prime}\right)$ for a fixed frequency of $f=1 \mathrm{~Hz}$ are shown against temperature for both longitudinal and transverse $\beta$-PVDF samples. As expected, $E^{\prime}$ is higher for the longitudinal sample, as the molecular alignment is higher along the direction of the mechanical tests. This mechanical anisotropy was already probed by conventional quasi-static experiments $[4,5]$, where longitudinal films were found to be stiffer, brittle and with a higher ultimate strain and lower strain at break than the transversal ones.

A relaxation process with a maximum in $\tan \delta$ is detected at $\mathrm{T} \sim 238 \mathrm{~K}$. This process, also detected by dielectric methods, has been labelled $\beta$ or $\alpha_{a}$. It has been assigned to the cooperative segmental motions within the main chains of the amorphous regions [6]. Dielectric relaxation spectroscopy 


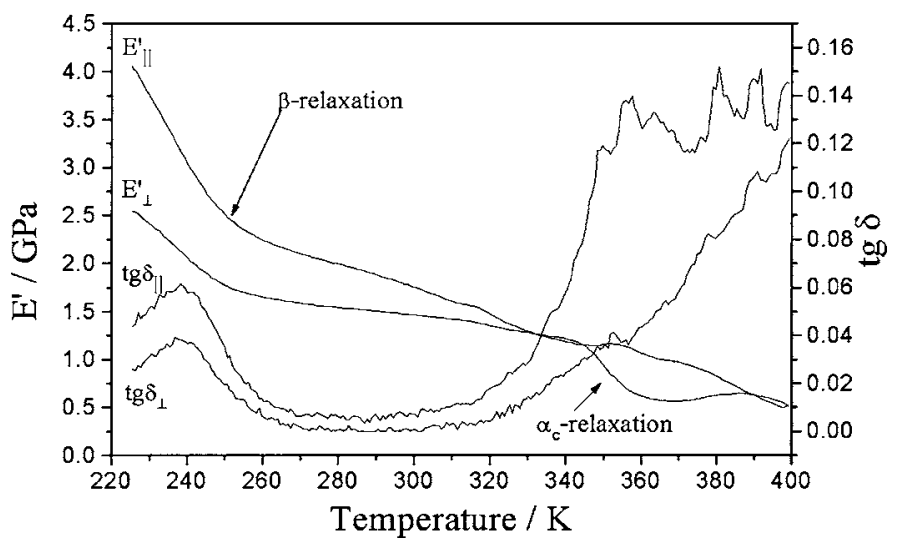

Figure 1. DMA results in longitudinal and transverse samples obtained at a frequency of $1 \mathrm{~Hz}$.

studies (Fig. 2) on the same film indicated that the central relaxation time of this process depends upon temperature according to the Vogel-TammanFulcher model [7], as it is observed in the relaxation assigned to the dynamical glass transition in amorphous and other semi-crystalline polymers. The damping associated with the $\beta$-process seems to be higher for the longitudinal sample. It indicates that the viscoelastic properties in anisotropic specimens depend strongly on the direction of the test [8]. Above $303 \mathrm{~K} \mathrm{a}$ new relaxation process emerges (Fig. 1), being more pronounced in terms of $\tan \delta$ for the longitudinal sample. This process, labelled $\alpha$ or $\alpha_{c}$, is absent in amorphous PVDF, and is associated with motions within the crystalline fraction $[3,6]$. This higher- $T_{g}$ relaxation is also found in a variety of flexible semicrystalline polymer [9]. It was suggested that this process involves rotational and translation mobility within the chains of the chain stems in the crystalline lamellae [9]. The significant cooperative character of this relaxation [10] indicates that diffusion processes involving chains in the amorphous region should accompany the $\alpha$-relaxation.

A direct comparison between the storage modulus $\left(\mathrm{E}^{\prime}\right)$ and the dielectric constant $\left(\varepsilon^{\prime}\right)$ and between the mechanical and dielectric loss factors $(\tan \delta)$ is shown in Fig. 2. The $\beta$-relaxation is identified by an increase of $\mathrm{E}^{\prime}$ and a corresponding decrease of $\varepsilon^{\prime}$ by lowering temperatures, i.e. an increase in the mechanical stiffness and a decrease in the dipole mobility with the applied field. The $\alpha$-relaxation, on the other hand, is only observed in the mechanical experiments, especially in the measurements performed parallel to the draw direction. $\varepsilon^{\prime}$ shows at that temperature a decrease that cannot be 


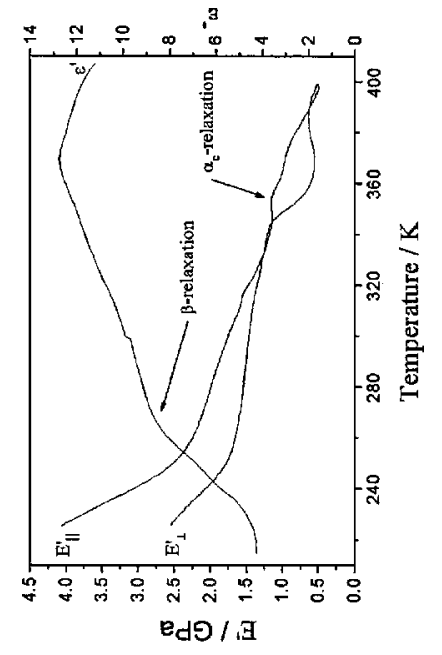

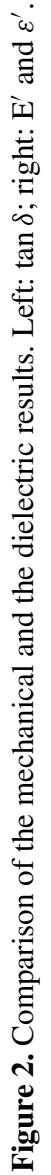

총 웅 음

8 क 
explained neither of the $\alpha$-relaxation, nor by a ferroelectric phase transition. This decrease in $\varepsilon^{\prime}$ is accompanied by an almost exponential increase in $\tan \delta$ observed both in the mechanical and the dielectric measurements (Fig. 2).

In fact, the mechanical experiments performed on the direction perpendicular to the preferred chain orientation and the dielectric measurements suffer the influence of an important geometrical effect that should be taken into account in order to address the properties of $\beta$-PVDF in the high temperature region. The comparison of $\tan \delta$ obtained both by mechanical and dielectric methods shows a similar behaviour, as stated previously: the low temperature $\beta$-relaxation and an increase of $\tan \delta$ starting at $\sim 334 \mathrm{~K}$ up to temperatures close to the melting point.

\subsection{Thermal Mechanical Analysis}

As referred earlier, there is an important geometrical effect in $\beta$-PVDF that severely affects the mechanical and dielectric measurements, as well as the performance of the material in different applications. This geometrical effect was measured by TMA experiments along the two main directions of the sample: parallel and perpendicular to the draw direction. Samples with and without silver electrodes were analysed in order to characterize the influence of the electrodes on the thermal expansion of the material, as it can be important for many applications.

As can be observed in Fig. 3, the samples have two different regimes for the thermal expansion: from room temperature to $\sim 364 \mathrm{~K}$ and from this temperature to $\sim 414 \mathrm{~K}$. From room temperature to $\sim 364 \mathrm{~K}$ the samples slightly increase perpendicularly to the draw direction and suffer a slight shrinking along the draw direction. It is interesting to note that the electrodes prevent this shrinking. At $\sim 364 \mathrm{~K}$ the geometrical effect begins to be more important: perpendicularly to the draw direction, the sample still increases linearly by increasing temperature but in a more pronounced way. On the other hand, the sample suffers a pronounced shrinkage along the draw direction [11]. As a result, the area of the sample undergoes a slight increase from room temperature to $\sim 364 \mathrm{~K}$ and shrinkage from this temperature to $\sim 414 \mathrm{~K}$ (Fig. $3)$.

In Fig. 3 the behaviour of both electroded and non-electroded samples is also depicted. It is worth noticing that the difference in the thermal expansion of electroded and non-electroded samples increases linearly, excepting for the transition region around $\sim 364 \mathrm{~K}$ (inset in Fig. 3). This difference must be obviously taken into account when evaluating the practical performance of the material. 


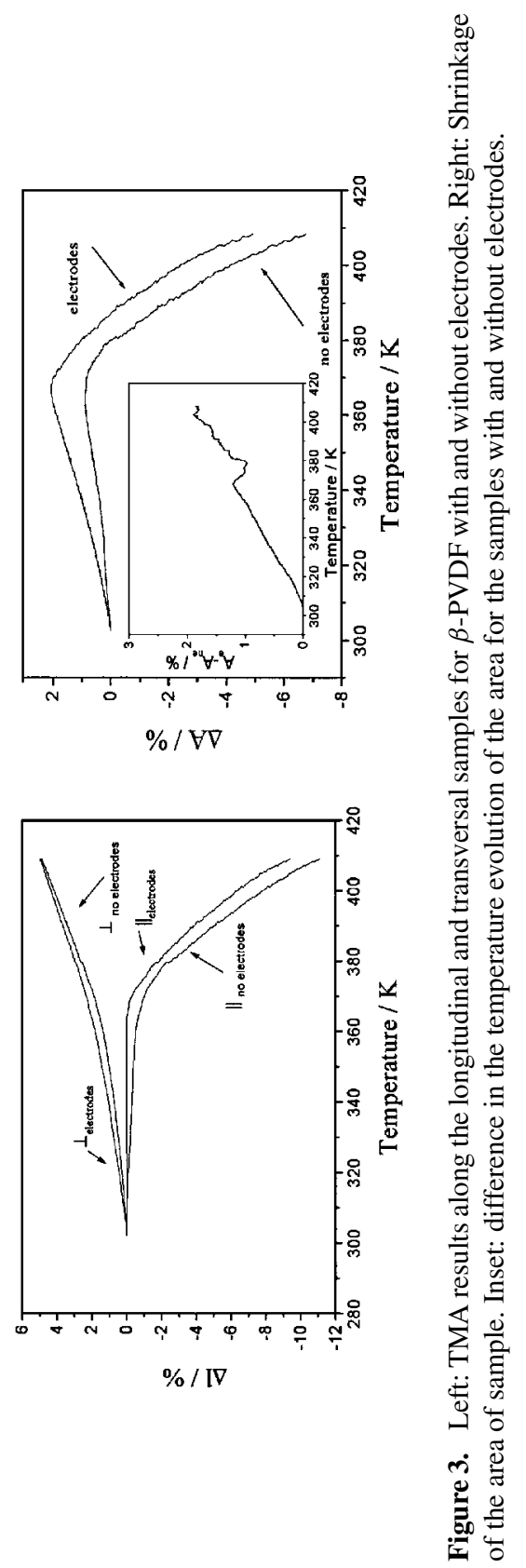


TABLE I Linear thermal expansion coefficients calculated from the TMA experiments

\begin{tabular}{lrrr}
\hline \multicolumn{1}{c}{ PVDF } & \multicolumn{1}{c}{$\lambda_{1}\left(\mathrm{~K}^{-1}\right)$} & \multicolumn{1}{c}{$\lambda_{2}\left(\mathrm{~K}^{-1}\right)$} \\
\hline Sample with silver electrodes & $\|$ & $6 \times 10^{-6}$ & $-2.56 \times 10^{-3}$ \\
& $\perp$ & $3.11 \times 10^{-4}$ & $6.8 \times 10^{-4}$ \\
Sample with no electrodes & $\|$ & $9.1 \times 10^{-5}$ & $-2.8 \times 10^{-3}$ \\
& $\perp$ & $2.27 \times 10^{-4}$ & $7.71 \times 10^{-4}$ \\
\hline
\end{tabular}

To quantify this effect, the thermal coefficient of linear expansion, $\lambda$ (Eq. 1) was calculated:

$$
\lambda=\frac{1}{L} \frac{d L}{d T} .
$$

Here, L stands for the length of the material along the desired direction. The results are summarized in Table I.

In order to better understand the significance of the shrinkage, it is necessary to understand how it affects the capacity of the sample [12]. The capacity of the polymer film capacitor follows the common capacitor formula (Eq. 2)

$$
C=\frac{\varepsilon_{o} \varepsilon_{r} A}{d},
$$

where $C$ is the capacitance, $\varepsilon_{r}$ is the dielectric constant of the system, $\varepsilon_{0}$ is the permittivity of free space, $A$ is the area of the sample and $d$ is the thickness. If $\mathrm{A}$ is reduced because of shrinkage, so too is the capacitance value. The important consequence that can be obtained from these results is that the geometrical variations above $364 \mathrm{~K}$ are big enough to be taken into account in any practical application.

At $\mathrm{T} \geq 345 \mathrm{~K}$ the frequency dependent of the real (imaginary) part of complex permittivity of $\beta$-PVDF can be fitted by Eq. 3 :

$$
\varepsilon^{*}(\nu)=\varepsilon_{\infty}+B \operatorname{Im}(i 2 \pi \nu)^{a},
$$

where $\varepsilon_{\infty}$ is the high frequency limit of the real part of the dielectric permittivity, which correspond to the static permittivity of the nonconductive crystal, $B$ and $a$ are constants. The obtained values $(0.10<a<0.25)$ are typical for hopping conduction in disordered media [13]. This effect is probably due to ionic species from chemical additives used for polymerization 
as well as dissociated water into the polymer. The fittings could not be performed properly for temperatures $\mathrm{T}>364 \mathrm{~K}$ due to the influence of the shrinkage of the sample.

\subsection{Infrared Spectroscopy}

The vibrational spectrum of PVDF has been well characterized and several absorption bands characterize the $\alpha$ - and $\beta$-phases of the polymer, the degree of crystallinity and the chain orientation $[5,7]$. In order to better understand the high temperature behavior of $\beta$-PVDF we have studied the changes in absorbance of some representative bands of $\beta$-PVDF after a heat treatment was carried out to the following temperatures for one minute: 334, 364, 394 and $414 \mathrm{~K}$ [14].

The effects of temperature on the infrared spectra are presented in Fig. 4. The absorbance of the bands was normalized to the mode at $677 \mathrm{~cm}^{-1}$. This absorption band points to the presence of head-to-head and tail-to-tail configurations [5]. This peak is observable in both polarization directions and is relatively not affected by the temperature changes. The absorbance values of this peak are taken as reference to calculate relative changes on the intensities of the other peaks, as the ratio of absorbances is not dependent on the sample thickness.

As a general result, whereas the absorbance of some relevant modes in parallel polarization follow a similar pattern to the one observed in the dielectric constant [14], i.e. the absorbance has a maximum at $\mathrm{T} \sim 364 \mathrm{~K}$, where
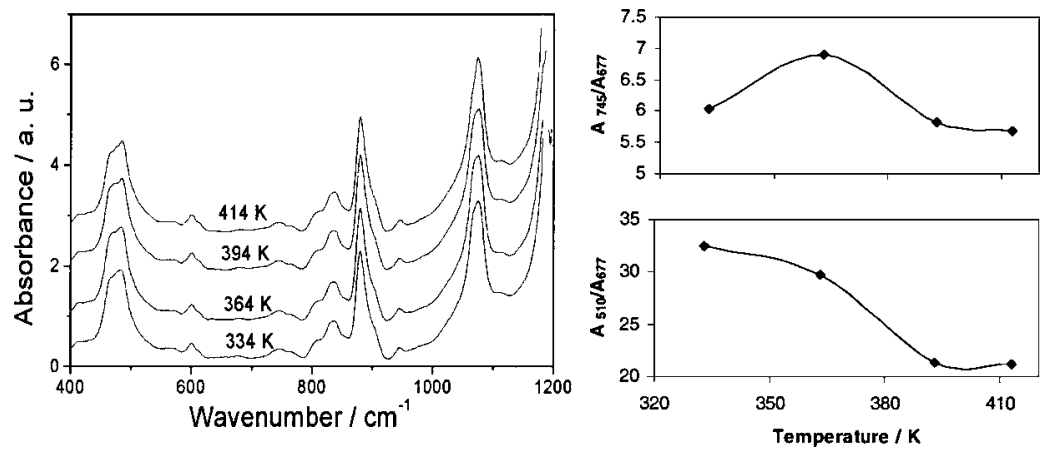

Figure 4. Left: comparison of the FTIR spectra for $\beta$-PVDF with four different temperature treatments $(334 \mathrm{~K}, 364 \mathrm{~K}, 394 \mathrm{~K}$ and $414 \mathrm{~K})$ in parallel mode. Right: Evolution of the normalized absorbance with the temperature treatment for the modes at $745 \mathrm{~cm}^{-1}$ (perpendicular polarization) and at $510 \mathrm{~cm}^{-1}$ (parallel polarization). 
$\varepsilon^{\prime}$ shows a maximum, the modes observed in the perpendicular polarization show a continuous decrease in the absorbance (Fig. 4). A strong reduction of the absorbance in both polarizations above $364 \mathrm{~K}$ indicates important conformational changes in the polymeric chains accompanying the shrinkage of the sample. The actual dipolar reorientation during heating the material is demonstrated by the curve of the infrared absorbance versus temperature for the $510 \mathrm{~cm}^{-1}$ band (Fig. 4). This mode is particularly important, as it is associated with vibrations along the direction of the $\mathrm{CF}_{2}$ dipole vector; this mode is perpendicular to the chain axis and parallel to the polar $b$-axis [7]. The band at $745 \mathrm{~cm}^{-1}$ is also characteristic of the $\beta$-phase and assigned to a rocking mode [5] (Fig. 4). When the film undergoes thermal exposure at elevated temperatures the molecular chains become mobile (trying to revert in to the tangled state). This releases the stresses that were locked in when the film was originally stretched causing shrinkage in unrestrained film.

\section{CONCLUSION}

The $\beta$ - and $\alpha$-relaxations have been identified in the DMA experiments performed along the main directions of the material. These relaxations determine the viscoelastic performance of the material at low and above room temperature, respectively. A comparison with the dielectric results indicates that the same molecular units contribute to the mechanical and the dielectric relaxation.

Two regimes in the thermal expansion of the material have been identified. The high temperature shrinkage deeply affects the mechanical and dielectric behaviour of $\beta$-PVDF. This shrinkage is due to the release of the stress that was locked in for the preparation of the $\beta$-form of PVDF.

\section{ACKNOWLEDGEMENT}

Work supported by the Portuguese Foundation for Science and Technology under Grant POCTI/CTM/33501/99.

\section{REFERENCES}

[1] L. M. Blinov, V. M. Fridkin, S. P. Palto, A. V. Bune, P. A. Downen, and S. Durcharme, Physiscs-Uspekhi 43, 243 (2000). 
[2] T. Furukawa, Phase Transitions 18, 143 (1989).

[3] A. J. Lovinger, Developments in Crystalline Polymers, Vol. 1 (Ed. D.C. Basset, London, Elsevier Applied Science, 1982).

[4] A. M. Vinogradov and F. Halloway, J. Adv. Mater. 29, 11 (1997).

[5] S. Lanceros-Mendez, J. F. Mano, A. M. Costa, and V. H. Schmidt, J. Macrom. Sci.-Phys. B40 517 (2001).

[6] D. K. Das-Gupta, Ferroelectrics 33, 75 (1981).

[7] S. Laceros-Mendez, M. V. Moreira, J. F. Mano, V. H. Schmidt, and G. Bohannan, Ferroelectrics 273, 15 (2002).

[8] H. Zhou and G. L. Wilkes, Macromolecules 30, 2412 (1997).

[9] J. F. Mano, V. Sencadas, A. Mello Costa, and S. Lanceros-Méndez, to appear in Mat. Eng. A.

[10] S. Laceros-Mendez, J. F. Mano, and J. A. Mendes, Ferroelectrics 270, 271 (2002).

[11] J. H. Vinson and B. J. Jungnickel, Ferroelectrics 216, 63 (1998).

[12] I. W. Clelland and R. A. Price, Proceedings of the 18th Annual Capacitor and Resistor Technology Symposium, (Illinois Tool Works Inc., 1998).

[13] J. C. Dyre and T. B. Schroder, Rev. Mod. Phys. 72, 3 (2000).

[14] S. Lanceros-Méndez, V. Sencadas, and J. F. Mano, to be published. 